\title{
Composición química y efecto antibacteriano in vitro de extractos de larrea tridentata, origanum vulgare, artemisa ludoviciana y ruta graveolens Chemical composition and antibacterian effect in vitro of extracts of larrea tridentata, origanum vulgare, artemisa ludoviciana and ruta graveolens
}

Lucía Delgadillo Ruíz ${ }^{1}$, Rómulo Bañuelos Valenzuela ${ }^{2}$, Olivia Delgadillo Ruíz ${ }^{3}$, Mónica Silva Vega $^{2}$ y Perla Gallegos Flores ${ }^{1}$

Palabras Clave: extractos; actividad antimicrobiana; bacterias; inhibición

Recepción: 14-06-17 / Aceptación: 09-07-17

\section{Resumen}

\section{Introducción}

Los extractos de algunas plantas han demostrado tener propiedades antimicrobianas relacionadas a ciertos compuestos químicos como son el timol, carvacrol, limoneno, linalol y terpineno. El objetivo del presente trabajo fue determinar la concentración de estos compuestos en los extractos de Larrea tridentata, Origanum vulgare, Artemisa ludoviciana y Ruta graveolens; así como evaluar su efecto antimicrobiano en Escherichia coli, Acinetobacter baumanii, Pseudomona sp y Staphylococcus aureus.

\section{Método}

Los extractos se obtuvieron por destilación simple empleando alcohol etílico como solvente, la composición química se evaluó mediante cromatografía de gases. La actividad antimicrobiana de cada uno de los extractos de plantas se realizó por los métodos difusión en pozo y difusión en disco.

\section{Resultados}

Las bacterias mostraron diferentes grados de sensibilidad a los extractos, presentando inhibición de crecimiento $S$. aureus con el extracto de $O$. vulgare y $R$. graveolens, mientras que la bacteria Pseudomona sp., con los extractos de A. ludoviciana, L. tridentata y $O$. vulgare.

\footnotetext{
${ }^{1}$ Laboratorio de Biotecnología, Unidad Académica de Ciencias Biológicas, Universidad Autónoma de Zacatecas. Email: apozolero@hotmail.com

${ }^{2}$ Unidad Académica de Medicina Veterinaria y Zootecnia, Universidad Autónoma de Zacatecas

${ }^{3}$ Campus San Luis Potosí, Colegio de Postgraduados

(C) Universidad De La Salle Bajío (México)
} 


\section{Discusión}

La mayor concentración de timol y carvacrol se encontró en los extractos de $O$. vulgare y $L$. tridentata. El compuesto linalol se encontró en una proporción mayor en $O$. vulgare y en menor proporción en A. ludoviciana. Limoneno se encontró en los extractos de $O$. vulgare y $R$. graveolens. De las cuatro plantas evaluadas, el extracto de L. tridentata fue mejor, debido a que presenta la mayor inhibición en comparación con los otros extractos; y con un efecto similar a los aceites empleados como control. La técnica de difusión en disco, permitió observar mejor los efectos inhibitorios de los extractos y los aceites sobre cada una de las bacterias empleadas en comparación con el método de difusión en pozo.

\section{Abstract \\ Introduction}

Plant extracts have been shown to have antimicrobial properties related to certain chemical compounds such as thymol, carvacrol, limonene, linalool and terpinene. The objective of this study was to determine the concentration of these compounds in the extracts of Larrea tridentata, Origanum vulgare, Artemisa ludoviciana and Ruta graveolens; as well as to evaluate the antimicrobial effect in Escherichia coli, Acinetobacter baumanii, Pseudomona sp and Staphylococcus aureus.

\section{Method}

The extracts were obtained by simple distillation using ethyl alcohol as solvent, the chemical composition was evaluated by gas chromatography. The antimicrobial activity of each of the plant extracts was performed by well diffusion and disk diffusion methods.

\section{Results}

The bacteria showed different degrees of sensitivity to the extracts, $S$. aureus inhibition of growth with the extract of $O$. vulgare and $R$. graveolens, while the bacterium Pseudomona sp. showed inhibition with the extract of A. ludoviciana, L. tridentate and O. vulgare. 


\section{Discussion}

The highest concentration of thymol and carvacrol was found in the extracts of $O$. vulgare and $L$. tridentata. The compound linalool was found in a higher proportion in $O$. vulgare and to a lesser extent in A. ludoviciana. Limonene was found in the extracts of $O$. vulgare and $R$. graveolens. Of the four plants evaluated, L. tridentata extract was better because it had the highest inhibition compared to the other extracts; and with an effect similar to the oils used as control. The disk diffusion technique allowed better observation of the inhibitory effects of the extracts and oils on each of the bacteria used in comparison to the well diffusion method.

Keywords: extracts; antimicrobial activity; bacteria, inhibition. 


\section{Introducción}

Los extractos de plantas han sido empleados por el hombre en el tratamiento de enfermedades bacterianas. Estos son mezclas complejas de metabolitos secundarios lipófilos volátiles. Son extraídos de las plantas con disolventes y destilación por arrastre de vapor (Burt, 2004). Factores ambientales, la etapa de crecimiento y la salud de la planta determinan las concentraciones de los extractos (Dudareva et al. 2004).

La sensibilidad de las bacterias a aceites esenciales no es constante, esta propiedad es de interés para los nutricionistas rumiantes por la aplicación en los cambios de la fermentación en el rumen a través de la selección a favor o en contra de grupos específicos de microorganismos (Benchaar y Greathead, 2011). Cowan (1999), Kalemba y Kunicka (2003) y Burt (2004) han reportado las propiedades antimicrobianas y fungicidas que tienen los aceites esenciales de plantas.

Los compuestos de estos aceites, como timol, carvacrol y monoterpenos fenólicos presentan actividad antimicrobiana y se encuentran en altas concentraciones en aceite de orégano (Helander et al. 1998; Ultee et al. 2000 a, b). Paparella et al. (2008) y Xu et al. (2008) demostraron que el aceite de orégano es eficaz para aumentar la fluidez y la permeabilidad de la membrana citoplasmática dando lugar a la pérdida del contenido celular y la lisis celular de los microorganismos.

En relación con L. tridentata o gobernadora se ha documentado que más de 45 bacterias son susceptibles a su resina o sus componentes, así como a diez levaduras, nueve hongos y tres parásitos intestinales que atacan a los humanos comprometiendo su estado de salud. Los compuestos presentes en la gobernadora como los flavonoides actúan contra virus que afectan el RNA, y que ocasionan graves enfermedades como polio, sida y herpes (Saldívar, 2003).

Algunas especies de Artemisia (A. absinthium, A. biennis, A. frigida y A. ludoviciana) han sido utilizadas como antisépticos en la medicina tradicional por los nativos de América del Norte debido a sus propiedades antimicrobianas y antioxidantes. Entre sus usos se puede mencionar el tratamiento de yagas, heridas e infecciones de pecho (Lopes-Lutz et al. 2008).

Pathak et al. (2003) y Pandey et al. (2011) mencionaron que el extracto hidro-alcohólico de hojas de Ruta graveolans puede ser usado como un potente antiparasitario, antioxidante y antimicrobiano. Pandey et al. (2012) demostraron que el extracto de R. graveolans puede ser usado para disminuir la incidencia y gravedad de diarreas en ratones.

No 19, Vol. 9 (2), 2017. ISSN 2007 - 0705, pp.: 273 - 290 
Se han encontrado serios problemas de resistencia de microorganismos a los medicamentos antimicrobianos, dentro de los cuales destaca Staphylococcus aureus, Escherichia coli y Pseudomonas aeruginosa, en especial en el ámbito hospitalario; mientras las infecciones por Staphylococcus no adquiridas en hospitales pueden tratarse con los antibióticos derivados betalactámicos, las infecciones adquiridas en hospitales son, en su gran mayoría, resistentes a estos antibióticos y requieren tratamientos alternativos más costosos y, muchas veces, de mayor toxicidad. La resistencia se extiende a antimicóticos, antimaláricos y antivirales, haciendo cada vez menos efectivos los viejos medicamentos (AHFS, 2005).

Este problema de la resistencia se ha extendido igualmente al campo de la terapéutica veterinaria pues el uso indiscriminado de antibióticos y a veces su adición en los concentrados usados en la alimentación de animales, ha determinado que muchas bacterias como E. coli hayan adquirido cada vez mayor resistencia.

La obtención de antibióticos a partir de hongos o desde la síntesis química ha disminuido sustancialmente en los últimos 30 años. La industria farmacéutica y la comunidad científica basados en los resultados del aprovechamiento de plantas superiores de uso tradicional para el cuidado de la salud están estimulando su uso como antibióticos. Diversos autores de países desarrollados en la síntesis química mencionan la importancia de recurrir a fuentes naturales, entre ellas las plantas, para explorar nuevas moléculas con actividad antiinfecciosa (Ramírez et al. 2013; González et al. 2017).

Por lo que, el objetivo de este estudio fue determinar la concentración de timol, carvacrol, linalol, terpinene y limoneno en los extractos de L. tridentata, O. vulgare, A. ludoviciana y $R$. graveolens; para evaluar su efecto antimicrobiano en E. coli, A. baumanii, Pseudomona sp y $S$. aureus por los métodos de difusión en pozo y difusión en disco.

\section{Método}

El estudio se realizó en el estado de Zacatecas, México. Se utilizaron plantas de la medicina tradicional L. tridentata, O. vulgare, A. ludoviciana y $R$. graveolens, comúnmente llamadas gobernadora, oregano, estafiate y ruda respectivamente. Fueron recolectadas de crecimiento silvestre durante primavera y verano de 2013-2014. Cada una de las plantas fue secada a temperatura ambiente durante al menos dos semanas, después fueron desecadas durante 24 horas 
en un horno convencional a $45^{\circ} \mathrm{C}$, para eliminar completamente la humedad, y fueron molidas y almacenadas en bolsas herméticas para procedimientos posteriores (López et al. 2005).

En cada extracción se utilizó etanol al 70\%. En frascos de color ámbar de un litro fueron colocados veinte gramos de la muestra molida por cada $300 \mathrm{~mL}$ de solvente. La muestra fue sellada y mezclada vigorosamente por 10 min. Se dejó macerar durante un mes, a temperatura ambiente agitando cada tres días la mezcla por $10 \mathrm{~min}$. El sobrenadante fue pasado a través de papel filtro (Whatman No. 2) para remover los restos del polvo de la planta y el solvente fue evaporado en un extractor tipo Soxhlet a $85^{\circ} \mathrm{C}$ (Pesewu et al. 2008). Se hicieron dos alícuotas; una de ellas fue utilizada para determinar la composición química del extracto y otra para evaluar la actividad antimicrobiana.

\section{Composición química del extracto por cromatografía de gases}

La composición química se determinó mediante el uso del cromatógrafo de gases (CG) Agilent Tecnologies serie 6890N empleando una columna polar DB_WAXetr. Las condiciones de trabajo fueron: temperatura después de la inyección $250^{\circ} \mathrm{C}$ a una presión de 12.13 psi con un flujo de He $36.5 \mathrm{~mL} / \mathrm{min}$. Las condiciones para la columna fueron: temperatura inicial $50^{\circ} \mathrm{C}$ de cero a dos minutos, aumentando $10^{\circ} \mathrm{C}$ hasta llegar a $250^{\circ} \mathrm{C}$, manteniendo la temperatura constante por cinco minutos para luego descender a $50^{\circ} \mathrm{C}$ por dos minutos con un flujo de $\mathrm{He}$ de $1.6 \mathrm{~mL} / \mathrm{min}$ a una presión de 12.13 psi y una velocidad promedio de $25 \mathrm{~cm} / \mathrm{seg}$. Utilizando un detector de ionización de flama (FID) a una temperatura de $210^{\circ} \mathrm{C}$ con un flujo de $\mathrm{H}_{2}$ de $40 \mathrm{~mL} / \mathrm{min}$ y un flujo de aire de $450 \mathrm{~mL} / \mathrm{min}$, permitieron cuantificar los compuestos en las muestras.

Cuadro 1. Concentraciones empleadas de los estándares para la cuantificación en el CG.

\begin{tabular}{cccccc}
\hline Estándar & Timol & Carvacrol & Linalol & Terpineno & Limoneno \\
& $(\mathbf{m g} / \mathbf{m l})$ & $(\mathbf{m g} / \mathbf{m l})$ & $(\mathbf{m g} / \mathbf{m l})$ & $(\mathbf{m g} / \mathbf{m l})$ & $(\mathbf{m g} / \mathbf{m l})$ \\
\hline 1 & 10.373 & 8.284 & 7.744 & 7.154 & 8.496 \\
2 & 5.186 & 4.142 & 3.872 & 3.577 & 4.248 \\
3 & 2.593 & 2.071 & 1.936 & 1.789 & 2.124
\end{tabular}


Composición química y efecto antibacteriano in vitro de extractos de larrea tridentata, origanum vulgare, artemisa ludoviciana y ruta graveolens

$\begin{array}{llllll}4 & 1.297 & 1.035 & 0.968 & 0.894 & 1.062 \\ 5 & 0.648 & 0.518 & 0.484 & 0.447 & 0.531 \\ 6 & 0.324 & 0.259 & 0.242 & 0.224 & 0.265\end{array}$

\section{Fuente de bacterias}

Se utilizaron bacterias gram negativas: E. coli, A. baumanii y Pseudomona sp., y una bacteria gram positiva: $S$. aureus, las cuales fueron identificadas por pruebas bioquímicas en paneles comerciales por el equipo Phoenix 100 Becton Dickinson and Company® Sparks, Maryland 21152 USA. A partir de las bacterias cultivadas en el agar nutritivo, fueron seleccionadas cuatro o cinco colonias de cada una y se diluyeron en solución salina, hasta alcanzar una concentración de $0.45^{\circ} \mathrm{McFarland}$ para E. coli, $0.56^{\circ} \mathrm{McFarland}$ A. baumanii, $0.54^{\circ} \mathrm{McFarland}$ Pseudomona sp., y $0.55^{\circ} \mathrm{McFarland} S$. aureus. Se realizó una siembra masiva sobre agar Mueller Hinton (Álvarez et al. 2005), se escogieron el CEDAX ceftibuten (36 mg/mL) como control positivo para E. coli, A. baumannii, Pseudomona sp. (Barriga et al. 2008) y Butimaxil dicloxacilina (50 $\mathrm{mg} / \mathrm{mL}$ ) para S. aureus (Paniagua et al. 2006). Además, se trabajó con dos aceites: O. vulgare y hojas de Cinnamomum zeylanicum (canela) utilizados como controles positivos por su actividad antimicrobiana comprobada en otros trabajos (Matos et al. 2010; Brito-Junior et al. 2012; RosasPiñón et al. 2012) y como control negativo agua destilada.

\section{Actividad antibacteriana}

La actividad antibacteriana de cada extracto se determinó por dos métodos: difusión en pozo (perforación en gel de agar) (Álvarez et al. 2005) y difusión en disco (método disco-placacultivo) (Albado et al. 2001). En el medio Mueller Hinton, ya solidificado, se hicieron cuatro perforaciones de $7 \mathrm{~mm}$ de diámetro, se sellaron con $20 \mu \mathrm{L}$ del mismo agar, para evitar la dispersión del extracto. Las cepas diluidas de la misma forma que en el antibiograma, se sembraron masivamente y, una vez realizado esto, a cada pozo se le adicionó $20 \mu \mathrm{L}$ de cada extracto y $20 \mu \mathrm{L}$ de agua destilada, como control negativo, se incubó a $37^{\circ} \mathrm{C}$, por 24 horas. En la difusión de disco, las cepas diluidas se sembraron masivamente sobre el agar y se colocaron en la superficie de los medios cuatro discos de papel de filtro (Whatman) de $7 \mathrm{~mm}$ de diámetro, impregnados con $20 \mu \mathrm{L}$ de cada uno de los extractos y agua destilada como control negativo, los 
cuales se incubaron a $37^{\circ} \mathrm{C}$ por 24 horas. Ambas pruebas se efectuaron por triplicado. Se midió el diámetro del halo de inhibición del crecimiento de los microorganismos y el cálculo del porcentaje del efecto inhibitorio relativo respecto al control positivo, se estimó aplicando la expresión (Martínez et al. 1996):

$$
\% \text { efecto inhibitorio }=\frac{\text { Media diámetro halo de inhibición }}{\begin{array}{c}
\text { Diámetro halo de inhibición } \\
\text { control positivo }
\end{array}} \times 100
$$

\section{Análisis estadístico}

Se aplicó ANOVA unifactorial; la prueba de Tukey, para comparar las medias entre más de dos grupos. La significancia, se reportó con un nivel de confianza del 99\% ( $\mathrm{p}=0.01)$. Para realizar estos cálculos, se empleó el programa estadístico SPSS 17.0.

\section{Resultados}

En el Cuadro 2 se muestra el volumen y el rendimiento de los extractos de L. tridentata, $O$. vulgare, A. ludoviciana y $R$. graveolens obtenidos. Los valores que se muestran representan el promedio de las repeticiones. El cuadro 3 muestra la composición química de cada uno de los extractos obtenidos por cromatografía de gases.

Cuadro 2. Rendimiento de los extractos de 20 gramos de cada una de las plantas.

\begin{tabular}{ccc}
\hline Muestra & Volumen extracto $(\mathbf{m L})$ & Rendimiento \% (W/W) \\
\hline L. tridentata & 19.43 & 6.475 \\
O. vulgare & 41.5 & 13.83 \\
A. ludoviciana & 17.95 & 5.98 \\
R. graveolens & 31.5 & 10.5 \\
\hline
\end{tabular}


Cuadro 3. Composición de los extractos mediante cromatografía de gases.

\begin{tabular}{|c|c|c|c|c|c|}
\hline Extractos & $\begin{array}{c}\text { Timol } \\
(\mathrm{mg} / \mathrm{mL})\end{array}$ & $\begin{array}{c}\text { Carvacrol } \\
(\mathrm{mg} / \mathrm{mL})\end{array}$ & $\begin{array}{l}\text { Linalol } \\
(\mathrm{mg} / \mathrm{mL})\end{array}$ & $\begin{array}{c}\alpha \text {-Terpineno } \\
(\mathrm{mg} / \mathrm{mL})\end{array}$ & $\begin{array}{r}\text { Limoneno } \\
(\mathrm{mg} / \mathrm{mL})\end{array}$ \\
\hline L. tridentata & 4.3033 & 7.7986 & ---- & ------- & ----- \\
\hline O. vulgare & 4.185 & 9.1027 & 12.5526 & ------- & 6.6491 \\
\hline A. ludoviciana & 2.5184 & 4.9087 & 2.886 & 5.1276 & ------ \\
\hline$R$. graveolens & 1.1626 & 2.8557 & ------- & --------- & 5.8022 \\
\hline
\end{tabular}

En los Cuadros 4 y 5 se muestran los resultados obtenidos de la medición del diámetro de halos de inhibición de los microorganismos estimados con las técnicas de difusión en pozo y en disco. Se utilizó como control positivo los aceites de orégano y canela ya que se ha reportado que presentan mayor efecto inhibitorio que un extracto por su composición química o sus compuestos activos (Duarte et al. 2005; Matos et al. 2010; Rosas-Piñón et al. 2012).

Cuadro 4. Efecto inhibitorio de los extractos mediante el método de disco.

\begin{tabular}{ccccc}
\hline \multirow{2}{*}{ Muestras } & \multicolumn{3}{c}{ Diámetro halos de inhibición (mm) } \\
\cline { 2 - 5 } & E. coli & A. baumanii & Pseudomona sp & S. aureus \\
Extracto L. tridentata & $17^{\mathrm{c}}$ & $19^{\mathrm{c}}$ & $15^{\mathrm{c}, \mathrm{d}}$ & $12^{\mathrm{b}, \mathrm{c}}$ \\
Extracto $O$. vulgare & $10^{\mathrm{d}}$ & $10.24^{\mathrm{d}}$ & $12.26^{\mathrm{d}, \mathrm{e}}$ & $15^{\mathrm{b}}$ \\
Extracto A. ludoviciana & $9^{\mathrm{d}}$ & $8^{\mathrm{d}}$ & $19^{\mathrm{b}, \mathrm{c}}$ & $8^{\mathrm{d}}$ \\
Extracto $R$. graveolens & $9^{\mathrm{d}}$ & $9^{\mathrm{d}}$ & $8^{\mathrm{e}}$ & $15.5^{\mathrm{a}, \mathrm{b}}$ \\
Aceite O. vulgare & $20.66^{\mathrm{b}}$ & $24.5^{\mathrm{c}}$ & $25^{\mathrm{b}}$ & $10.5^{\mathrm{c}, \mathrm{d}}$ \\
Aceite de hoja de C. zeylanicum & $23^{\mathrm{b}}$ & $21^{\mathrm{c}, \mathrm{b}}$ & $24.5^{\mathrm{b}}$ & $15^{\mathrm{b}}$ \\
CEDAX ceftibuten & $35.5^{\mathrm{a}}$ & $33^{\mathrm{a}}$ & $41^{\mathrm{a}}$ & $19^{\mathrm{a}}$ \\
Butimaxil dicloxacilina & & & & \\
\hline
\end{tabular}

Diferentes letras sobre las columnas de la tabla indican diferencias estadísticas significativas. $(\mathrm{p}<=0.01)$. 
Cuadro 5. Efecto inhibitorio de los extractos mediante el método de pozos.

\begin{tabular}{|c|c|c|c|c|}
\hline \multirow{2}{*}{ Muestras } & \multicolumn{4}{|c|}{ Diámetro halos de inhibición (mm) } \\
\hline & E. coli & A. baumanii & Pseudomona sp & S. aureus \\
\hline Extracto L. tridentata & $7^{\mathrm{c}}$ & $13^{\mathrm{b}, \mathrm{c}}$ & $19^{\mathrm{c}}$ & $7^{\mathrm{b}}$ \\
\hline Extracto $O$. vulgare & $13^{\mathrm{c}, \mathrm{b}}$ & $9^{c}$ & $23^{\mathrm{c}, \mathrm{d}}$ & $13^{\mathrm{a}}$ \\
\hline Extracto A. ludoviciana & $7^{\mathrm{c}}$ & $8^{c}$ & $7^{\mathrm{d}}$ & $7^{\mathrm{b}}$ \\
\hline Extracto $R$. graveolens & $7^{\mathrm{c}}$ & $9^{c}$ & $7^{\mathrm{d}}$ & $13^{\mathrm{a}}$ \\
\hline Aceite $O$. vulgare & $21^{\mathrm{b}, \mathrm{a}}$ & $7^{\mathrm{c}}$ & $27^{\mathrm{b}}$ & $7^{\mathrm{b}}$ \\
\hline Aceite de hoja de $C$. zeylanicum & $15^{\mathrm{b}, \mathrm{c}}$ & $19^{\mathrm{b}}$ & $7^{\mathrm{d}}$ & $7^{\mathrm{b}}$ \\
\hline CEDAX ceftibuten & $29^{a}$ & $45^{\mathrm{a}}$ & $37^{\mathrm{a}}$ & ---- \\
\hline Butimaxil dicloxacilina & ---- & ---- & ----- & $15^{\mathrm{a}}$ \\
\hline
\end{tabular}

Diferentes letras sobre las columnas de la tabla indican diferencias estadísticas significativas $(\mathrm{p}<=0.01)$.

En cuanto a la actividad antimicrobiana, se confirma que el extracto del $O$. vulgare, posee efecto antimicrobiano frente a bacterias gram positivas como $S$. aureus y sobre las bacterias gram negativas E. coli, A. baumanii y Pseudomona sp, mientras que el extracto de A. ludoviciana posee el mayor porcentaje de inhibición frente a Pseudomona sp., utilizando el método de disco, siendo diferente por el método de pozos donde el mayor porcentaje de inhibición se presenta en $S$. aureus efecto antimicrobiano en $A$. baumanii, por último el extracto de $R$. graveolens presentó mayor efecto antimicrobiano para S. aureus.

El porcentaje de efecto inhibitorio estimado mediante la ecuación 1, para el extracto de $L$. tridentata fue mayor para $S$. aureus por el método de difusión en disco; mientras que por el método de difusión en pozos fue mayor para Pseudomona. El extracto de $O$. vulgare y $R$. graveolens empleando los dos métodos, presentan mayor efecto inhibidor a la bacteria $S$. aureus. Finalmente, el extracto de A. ludoviciana por el método de difusión de disco presenta el mayor efecto inhibidor a la bacteria Pseudomona (Figura 1). 
Composición química y efecto antibacteriano in vitro de extractos de larrea tridentata, origanum vulgare, artemisa ludoviciana y ruta graveolens
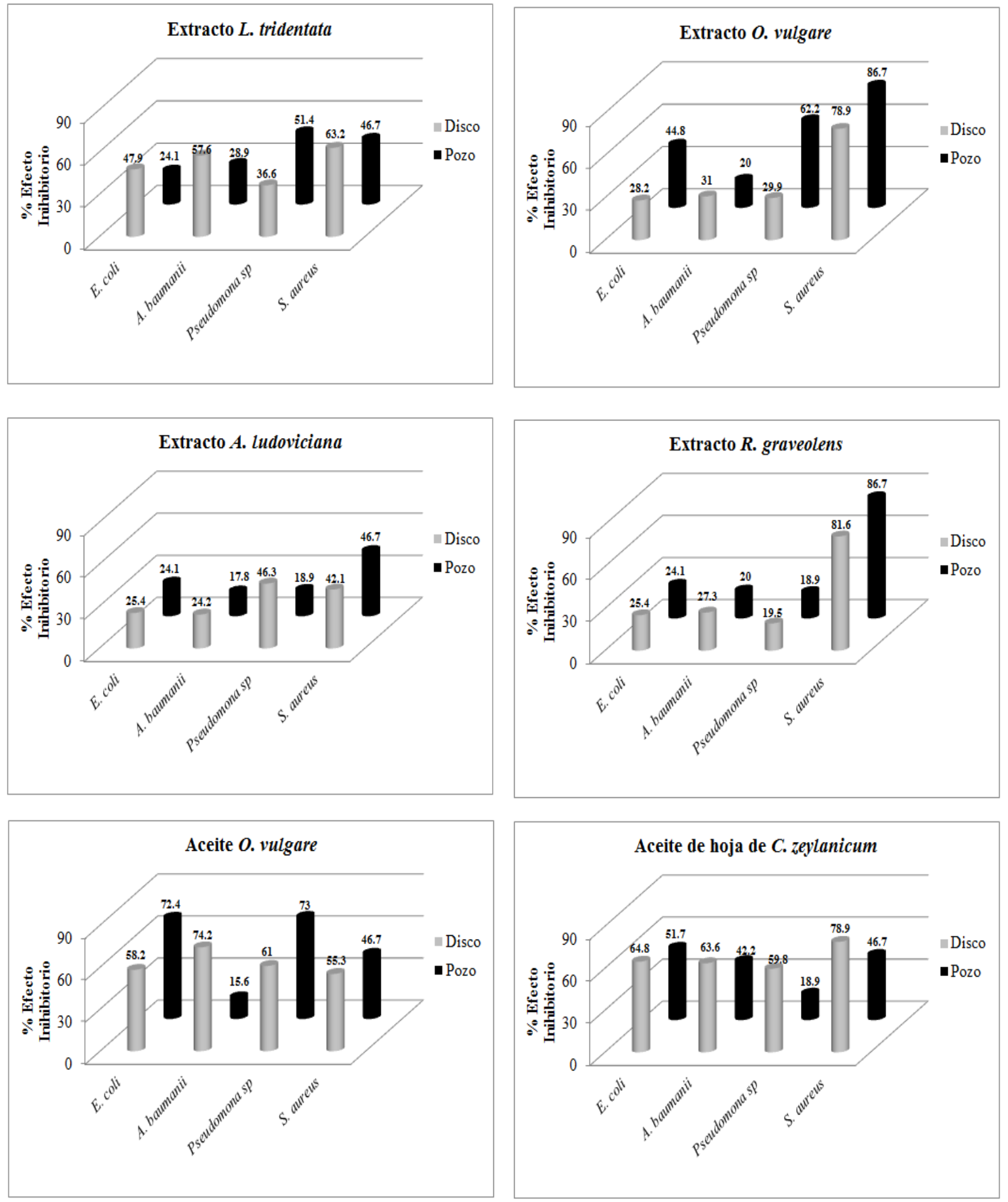

Figura 1. Porcentaje de efecto inhibitorio de los diferentes extractos y aceites. 


\section{Discusión o Conclusiones}

La presencia de metabolitos secundarios en las plantas ofrece la posibilidad de encontrar moléculas bioactivas que tengan actividad antimicrobiana, estas son muy promisorias y buscadas afanosamente, en especial por el alarmante incremento de la resistencia bacteriana (Mukherjee, 2002).

Martins et al. (2013) reportan en su estudio que en extractos de L. tridentata contienen compuestos bioactivos del tipo fenólicos como los flavonoides, quercetina, keampferol y ácido nordihidroguaiarético, diferentes a los reportados en este trabajo. El extracto de O. vulgare está compuesto por limoneno, linalol, carvacrol y timol. Estos resultados coinciden con los reportados por Albado et al. (2001) quienes analizaron aceites de orégano e identificaron terpineoles, fenoles y compuestos relacionados metabólicamente con el carvacrol.

En la composición química del extracto de A. ludoviciana se encontró terpineno, linalol, timol y carvacrol. Estos resultados difieren de lo reportado por Kordali et al. (2005), ellos encontraron que el extracto A. ludoviciana contiene principalmente anetol, beta-ocimeno, limoneno y metileugenol, ninguno de estos compuestos fueron identificados en este estudio ya que se debe tener presente que los principios activos dependen de factores genéticos, agronómicos y ambientales de las plantas.

De Feo et al. (2002) reportan que la composición química del aceite esencial $R$. graveolans está integrada por terpenoides que constituyen $11.2 \%$ del aceite, identificando $\alpha$ pineno, limoneno y 1,8-cineole como los principales constituyentes. Coincidiendo con los resultados obtenidos en la determinación de limoneno presente en $R$. graveolans.

Los constituyentes químicos: carvacrol, timol, terpineno, limoneno y linalol; dependiendo del origen y tipo de planta posen efectos antimicrobianos, antifúngicos y antioxidantes (Blazer, 2002). Acciones antimicrobianas se le atribuyen principalmente al carvacrol y timol.

Los resultados del efecto antimicrobiano del extracto de $O$. vulgare frente a E. coli y $A$. baumanii coincide con lo reportado por Aligiannis et al. (2001), ellos también encontraron que los aceites escenciales de la especie Origanum presentan esta actividad inhibitoria en bacterias gram negativas.

El preciso mecanismo de acción antibacteriana de los extractos de plantas y de sus compuestos bioactivos aún no han sido plenamente establecidos, sin embargo, se conoce que ocasionan cambios en la composición de los ácidos grasos de la membrana celular bacteriana; 
estos cambios han sido observados cuando las células son expuestas a concentraciones elevadas de los biocompuestos presentes en los extractos (Di Pascua et al. 2006). El carvacrol y timol dañan la membrana exterior de las bacterias gram negativas e incrementan la permeabilidad de la membrana citoplasmática que causa pérdidas de ATP, fuga de iones y lisis celular (Gill y Holley, 2006).

La composición química, las actividades antimicrobianas y antioxidantes de $A$. ludoviciana han sido analizadas por sus efectos inhibitorios sobre el crecimiento de bacterias y hongos. En relación con la composición química, en la literatura especializada se reporta que el extracto de A. ludoviciana contiene principalmente anetol (81\%), beta-ocimeno (6.5\%), limoneno (3.0\%) y metileugenol (1.8\%) (Kordali et al. 2005).

Sin embargo, en los resultados de está investigación, solo se observa el efecto inhibitorio a $S$. aureus, bacteria gram positivo al extracto A. ludoviciana; las bacterias gram negativas como E. coli y A. baumanii no presentan el efecto inhibitorio al extracto A. ludoviciana. De acuerdo con Yun et al. (2008), la ubicación de la planta y la estación de crecimiento influyen en la actividad antimicrobiana en la especie Artemisia orientalis, por lo que estos aspectos deben ser considerados.

Burt et al. (2007) señalaron que bacterias de $E$. coli crecen en presencia de carvacrol a una concentración de $0.05 \mathrm{mg} / \mathrm{mL}$ sin síntesis de flagelos, provocando que el microorganismo crezca sin movilidad, es decir, cuando la célula bacteriana está sujeta a un estrés ocasionado por sustancias toxicas y está en riesgo su viabilidad, la bacteria es capaz de suprimir funciones secundarias, como la formación del flagelo, y así conservar energía para otras funciones primordiales en la célula; este mecanismo de reacción bacteriana puede por lo tanto ser una táctica de supervivencia.

En relación con los extractos alcohólicos de $R$. graveolens, se encontró que tienen efecto inhibidor contra E. coli. El mismo resultado se observó sobre $S$. aureus que coincide por lo reportado por Castro et al. (2011).

Los resultados del presente trabajo permiten afirmar que los extractos de L. tridentata, $O$. vulgare, A. ludoviciana y $R$. graveolens son productores de sustancias bioactivas con efecto antibacteriano contra Staphylococcus aureus. Estas plantas se sumarían a las ya reportadas en la literatura científica como especies promisorias por su potencial bioactivo frente a dicha bacteria 
(Arango et al. 2004; Nasar y Halkman, 2004) en una intensa y ansiosa búsqueda de antibióticos obtenibles de plantas.

De los cuatro extractos de plantas evaluados, el extracto de L. tridentata fue mejor debido a que presenta la mayor inhibición en comparación con los otros extractos; y con un efecto similar a los aceites empleados como control. La mayor concentración de timol y carvacrol se encontró en los extractos de $O$. vulgare y L. tridentata. En $O$. vulgare además se encontró la presencia de linalol y limoneno pero su efecto inhibidor fue menor en comparación con el extracto de $L$. tridentata. Es probable que parte de la efectividad antibacteriana de L. tridentata se atribuya no solo a timol y carvacrol, posiblemente otros compuestos no identificados en este estudio estén influyendo (Marttins et al. 2013). El terpineno solo se encontró en A. ludoviciana. Mientras que concentraciones de limoneno se encontraron en los extractos de $O$. vulgare y $R$. graveolens.

La técnica de difusión en disco, permitió observar mejor el efecto inhibitorio de los extractos y los aceites sobre cada una de las bacterias empleadas (E. coli, A. baumanii, Pseudomona sp y S. aureus) en comparación con el método de difusión en pozo. Tomando como referencia el valor del diámetro del halo presente en la difusión de disco, es decir, a mayor diámetro de halo mayor será el efecto inhinitorio (como en este caso).

Los resultados obtenidos contribuyen al desarrollo de nuevas investigaciones relecionadas con el efecto antimicrobiano in vivo e in vitro de más bacterias ya sea de origen hospitalario o en el área de ciencias pecuarias. Por ejemplo, el uso de los extractos de plantas en la modificación de la fermentación ruminal que permita eficientizar el proceso de digestión, obteniendo mayor concentración de ácidos grasos volátiles, disminución de metano y dióxido de carbono (principales gases de efecto invernadero).

\section{Referencias}

AHFS (ED). (2005). Drug Information. American Society of Health System Pharmacists. Pp. 48887.

Albado, Emilia P; Saez, Gloria F. y Grabiel, Sandra A. (2001). Composición química y actividad antibacteriana del aceite esencial del Origanum vulgare (orégano). Rev. Med. Hered. 12: 16-19. 
Álvarez, María L.; Isaza, Gustavo M. y Echeverry, Harold L. (2005). Efecto Antibacteriano in vitro de Austroeupatorium inulaefolium H.B.K. (Salvia amarga) y Ludwigia polygonoides H.B.K. (Clavo de laguna). Biosalud. 14: 46-55.

Aligiannis, N.; Kalpotzakis, E.; Mitaku, S., and Chinou I.B. (2001). Composition and antimicrobial activity of the essential oils of two Origanum species. J. Agric. Food Chem. 49:4168-4170.

Arango, A. M., Sánchez, J. B., and Galvis, L. B. (2004). Productos naturales con actividad antimicótica. Rev. Esp. Quimioterap, 17(4), 325-331.

Barriga, A. G., González, N. F. M., \& Arumir, E. C. (2008). Susceptibilidad antimicrobiana in vitro de 1200 microorganismos Gram negativos causales de infecciones de vías urinarias. Enf. Inf. Microbiol, 28(3), 90-98.

Benchaar, Chaouki and Greathead, Henry. (2011). Essential oils and opportunities to mitigate enteric methane emissions from ruminants. Anim. Feed Sci. Technol. 166-167: 338-355.

Blazer, V. S. (2002). Histopathological assessment of gonadal tissue in wild fishes. Fish Physiology and Biochemistry, 26(1), 85-101.

Brito-Júnior, Manoel, Nobre, Sergio A., Freitas, Juliana C., Camilo, Carla C. and Faria-e-Silva, André L. (2012). Antibacterial activity of a plant extract and its potential for disinfecting gutta-percha cones. Acta Odontológica Latinoamericana, 25(1), 9-13.

Burt, Sara. (2004). Essential oils: their antibacterial properties and potential applications in foods_-a review. Int. J. Food Microb. 94: 223-253.

Burt, Sara, Zee, Ruurd, Koets, Ad., de Graaff, Anko, van Knapen, Frans, Gaastra, Wim., Haagsman, Henk and Veldhuizen, Edwin. 2007. Carvacrol Induces Heat Shock Protein 60 and Inhibits Synthesis of Flagellin in Escherichia coli O157:H7. Applied and Environmental Microbiology, 73 (14): 4484-4490.

Castro, Américo J.L.; Juárez, José R.E.; Ramos, Norma J.C.; Suárez, Silvia C.; Retuerto, Fernando P. y Gonzales, Sixto A.E. (2011). Structural elucidation of essential oil of Ruta graveolens L. Ruda, antioxidant activity and cytotoxicity bio-assay. Ciencia e Investigación. 14(1): 25-28.

Cowan, Marjorie M. (1999). Plant products as antimicrobial agents. Clin. Microbiol. Rev. 12: $564-582$. 
De Feo, V., F. De Simone and F. Senatore. (2002). Potential allele chemicals from the essential oil of Ruta graveolens. Phytochemistry. 61, 573-578.

Di Pasqua, Rosangela, Hoskins, Nikki, Betts, Gail and Mauriello, Gianluigi. (2006). Changes in membrane fatty acids composition of microbial cells induced by addiction of thymol, carvacrol, limonene, cinnamaldehyde, and eugenol in the growing media. Journal of agricultural and food chemistry, 54(7), 2745-2749.

Duarte, Marta C. T., Figueira, Glyn M., Sartoratto, Adilson, Rehder, Vera L. G. and Delarmelina, Camila. (2005). Anti-Candida activity of Brazilian medicinal plants. Journal of ethnopharmacology, 97(2), 305-311.

Dudareva, Natalia; Pichersky, Eran and Gershenzon, Jonathan. (2004). Biochemistry of plant volatiles. Plant Physiol. 135; 1893-1902.

Gill, A. O. and Holley, R. A. (2006). Inhibition of membrane bound ATPases of Escherichia coli and Listeria monocytogenes by plant oil aromatics. International journal of food microbiology, 111(2), 170-174.

González, Rafael. P., Sánchez, Yurisnel. O., Rivera, Rodisnel. P., Mompié, Saray. A. and Ginarte, Marta. L. H. (2017). 07-Relación de las propiedades físico-químicas con la actividad farmacológica de Zuedania guidonia (guaguasí) Relationship of the physicalchemical properties with the pharmacological activity of Zuedania guidonia (guaguasí). MULTIMED Revista Médica Granma, 19(4): 76-87.

Helander, Ilkka M.; Alakomi, Hanna L; Latva, Kyösti K.; Mattila, Tiina S.; Pol, Irene; Smid, Eddy J.; Gorris, Leon G.M. and Wright, Atte von. (1998). Characterization of the action of selected essential oil components on Gram negative bacteria. J. Agric. Food Chem. 46: 3590-3595.

Kalemba D. and Kunicka A. (2003). Antibacterial and antifungal properties of essential oils. Curr. Med. Chem. 10: 813-829.

Kordali, Saban; Cakir, Ahmet; Mavi, Ahmet; Kilic, Hamdullah and Yildirim, Ali. (2005). Screening of chemical composition and antifungal and antioxidant activities of the essential oils from three Turkish Artemisia species. J. Agric Food Chem 53: 1408-1416.

Lopes-Lutz, Daíse, Alviano, Daniela S., Alviano, Celuta S. and Kolodziejczyk, Paul P. (2008). Screening of chemical composition, antimicrobial and antioxidant activities of Artemisia essential oils. Phytochemistry, 69(8), 1732-1738. 
López, Alvin J., García, Aura M. and Rojas, Jhon. J. (2005). Evaluación de dos metodologías para determinar la actividad antimicrobiana de plantas medicinales. Boletín Latinoamericano y del Caribe de plantas medicinales y aromáticas.

Martins, Silvia, Teixeira, José A. and I. Mussatto Solange. (2013). Solid-state fermentation as a strategy to improve the bioactive compounds recovery from Larrea tridentata leaves. Appl. Biochem. Biotechnol. 171: 1227-1239.

Martínez, María J.; Betancourt, José B. y Alonso, Nancy G. (1996). Ausencia de actividad antimicrobiana de un extracto acuoso liofilizado de Áloe vera (sábila). Rev. Cubana Plant Med. 1(3):18-20. Doi: 10.1007/s12010-013-0222-2

Matos Chamorro, R. A., Quispe Condori, S., Quito Vidal, M. R. y Beltrán Cárdenas, S. K. (2010). Evaluación de la capacidad antimicrobiana del aceite esencial de orégano (Origanum vulgare) microencapsuladas en $\beta$-ciclodextrina aplicados en cultivos microbianos. Revista de Investigación en Ciencia y Tecnología de Alimentos, 1(1).

Mukherjee, P. K. (2002). Quality control of herbal drugs: an approach ro evaluation of botanicals. New Delhi: Business Horizons Publication.

Nasar-Abbas, S. M. and Halkman, A. K. (2004). Antimicrobial effect of water extract of sumac (Rhus coriaria L.) on the growth of some food borne bacteria including pathogens. International journal of food microbiology, 97(1), 63-69.

Pandey, Pinkee, Mehta, Archana., Hajra, Subhadip., John, Jinu. and Mehta, Pradeep. (2011). Antioxidant property, total Phenolic content and inhibition of $\alpha$-amylase activity of Ruta graveolens L. leaves extract. J. Pharm. Res, 4, 1735-1737.

Pandey, Pinkee, Mehta, Archana and Hajra, Subhadip. (2012). Antidiarrhoeal activity of ethanolic extracts of Ruta graveolens leaves and stem. Asian J Pharm Clin Res, 5(4), 65-68.

Paniagua, C. G. L., Monroy, P. E., Alonso T. J., Vaca, P. S., Negrete, A. E. and Pineda, O. J. (2006). Prevalencia de infecciones en herida quirúrgica en pacientes dados de alta de un hospital general. Rev. Med. Hosp. Gen. México, 69(2), 78-83.

Paparella, Antonello; Taccogna, Lorenzo; Aguzzi, Irene; Chaves, Clemencia L.; Serio, Annalisa; Marsilio Fulvio and Suzzi, Giovanna. (2008). Flow cytometric assessment of the antimicrobial activity of essential oils against Listeria monocytogenes. Food Control. 19: 11741182. 
Pathak, Sen, Multani, Asha S., Banerji, Pratip and Banerji, Prasanta. (2003). Ruta 6 selectively induces cell death in brain cancer cells but proliferation in normal peripheral blood lymphocytes: A novel treatment for human brain cancer. International journal of oncology, 23(4), 975-982.

Pesewu, George A., Cutler, Ronald. R. and Humber, David. P. (2008). Antibacterial activity of plants used in traditional medicines of Ghana with particular reference to MRSA. Journal of ethnopharmacology, 116(1), 102-111.

Ramírez, Alexander C., Isaza, Gustavo M. and Pérez, Jorge E. C. (2013). Vegetal species studied by their antimicrobial, immunomodulatory and hypoglicemic properties in CaldasColombia, Southamerica. Biosalud, 12(1), 59-82.

Rosas-Piñón, Yazmín, Mejía, Alicia, Díaz-Ruiz, Gloria, Aguilar, María I., Sánchez-Nieto, Sobeida, and Rivero-Cruz, J. Fausto. (2012). Ethnobotanical survey and antibacterial activity of plants used in the Altiplane region of Mexico for the treatment of oral cavity infections. Journal of ethnopharmacology, 141(3), 860-865.

Saldívar, Ricardo H. L. (2003). Estado actual del conocimiento sobre las propiedades biocidas de la gobernadora [Larrea tridentata (DC) Coville]. Revista Mexicana de Fitopatología, 21(2), 214-222.

Ultee, Annemieke; Kets, Edwin P.W.; Alberda, Mark; Hoekstra, Folkert A. and Smid, Eddy J. (2000 a). Adaptation of the food-borne pathogen Bacillus cereus to carvacrol. Arch. Microbiol. 174: 233-238.

Ultee, A.; Slump, R.A.; Steging, G. and Smid E.J. (2000 b). Antimicrobial activity of carvacrol toward Bacillus cereus on rice. J. Food Prot. 63: 620-624.

Xu, J.; Zhou, F.; Ji, B.P.; Pei, R.S. and Xu, N. (2008). The antibacterial mechanism of carvacrol and thymol against Escherichia coli. Lett. Appl. Microbiol. 47: 174-179.

Yun, Kyeong W.; Jeong, Hyung J. and Kim, Jong H. (2008). The influence of the growth season on the antimicrobial and antioxidative activity in Artemisia princeps var. orientalis. Industrial Crops and Products. 27: 69-74. 